\title{
Paula T. Hammond selected for 2019 David Turnbull Lectureship Award
}

$\mathrm{T}$ he Materials Research Society's (MRS) David Turnbull Lectureship Award recognizes the career of a scientist who has made outstanding contributions to understanding materials phenomena and properties through research, writing, and lecturing, as exemplified by the late David Turnbull of Harvard University. Paula T. Hammond, Massachusetts Institute of Technology (MIT), has been selected to give the Turnbull Lecture at the 2019 MRS Fall Meeting in Boston. She is cited "for her contributions to the science, engineering and applications of self-assembled macromolecular systems."
Hammond is the David H. Koch Chair Professor of Engineering at MIT and Head of the Department of Chemical Engineering. She is a member of MIT's Koch Institute for Integrative Cancer Research, the MIT Energy Initiative, and a founding member of the MIT Institute for Soldier Nanotechnologies. Her research in nanomedicine encompasses the development of biomaterials to enable drug delivery from surfaces with spatiotemporal control. She investigates novel responsive polymer architectures for targeted nanoparticle drug and gene delivery, and has developed self-assembled materials systems for electrochemical energy devices.
Hammond was elected into the National Academy of Sciences in 2019, the National Academy of Engineering in 2017, the National Academy of Medicine in 2016, and into the 2013 Class of the American Academy of Arts \& Sciences. She won the American Chemical Society Award in Applied Polymer Science in 2018, the 2013 AIChE Charles M. A. Stine Award, and the 2014 AIChE Alpha Chi Sigma Award for Chemical Engineering Research. She received her bachelor's and $\mathrm{PhD}$ degrees in chemical engineering from MIT.

Hammond has designed multilayered nanoparticles to deliver a synergistic combination of siRNA or inhibitors with chemotherapy drugs in a staged manner to tumors, leading to significant decreases in tumor growth and a great lowering of toxicity. She has published more than 320 papers and has filed more than 20 patent applications. She is the co-founder and member of the Scientific Advisory Board of LayerBio, Inc. and is a member of the Scientific Advisory Board of Moderna, Inc.

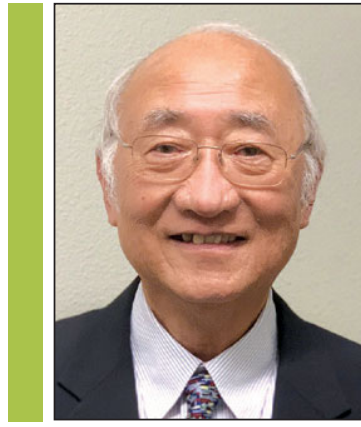

$\mathrm{T}$ he Materials Research Society (MRS) has named Lu Sham, University of California, San Diego, as the recipient of the 2019 Materials Theory Award "for pioneering contributions to the quantum theory of molecules and solids, especially the Kohn-Sham formulation of density functional theory." Sham will be recognized at the 2019 MRS Fall Meeting in Boston. The Materials Theory Award, endowed by Toh-Ming Lu and Gwo-Ching Wang, "recognizes exceptional advances made by materials theory to the funda- mental understanding of the structure and behavior of materials."

Sham is a Distinguished Professor Emeritus at the University of California, San Diego. He is a condensed-matter theorist, working on electron and phonon properties and quantum optical processes in semiconductor nanostructures and ferromagnets. Sham is an early practitioner of first-principles computations of lattice vibration spectra and density functional theory (DFT). His recent interests involve quantum information and spintronics.
Sham is a member of the National Academy of Sciences, and Academia Sinica, Taiwan, and a Fellow of the American Physical Society (APS), The Optical Society and American Association for the Advancement of Science. He served as a chair of the Semiconductor Commission of the International Union of Pure and Applied Physics, as a member of the Council and of the Executive Board of APS, and on the Executive Committee of its Division of Condensed Matter Physics. He received his bachelor's degree in mathematics from Imperial College London and his $\mathrm{PhD}$ degree in physics from the University of Cambridge.

The title of his talk Tuesday evening, December 3, is "Quantum Aspect of the Density Functional Theory." The KohnSham equation is a subterfuge from the classical nature of the DFT. Sham will give a brief description of modification of DFT to treat entanglement and to apply it to quantum phase transitions. 